\title{
Association between calcium in cord blood and newborn size in Bangladesh
}

\author{
Mariko Doi ${ }^{1 *}$, Rokeya Sultana Rekha ${ }^{2}$, Sultan Ahmed ${ }^{2}$, Masafumi Okada ${ }^{1}$, Anjan Kumar Roy ${ }^{2}$,
} Shams El Arifeen ${ }^{3}$, Eva-Charlotte Ekström ${ }^{4}$, Rubhana Raqib ${ }^{2}$ and Yukiko Wagatsuma ${ }^{1}$

${ }^{1}$ Department of Epidemiology, Graduate School of Comprehensive Human Sciences, University of Tsukuba, 1-1-1 Tennodai, Tsukuba, Ibaraki, Japan

${ }^{2}$ Nutritional Biochemistry Laboratory, Laboratory Science Division, International Centre for Diarrhoeal Disease Research, Bangladesh (ICDDR,B), GPO Box 128, Dhaka 1000, Bangladesh

${ }^{3}$ Child Health Unit, Public Health Division, International Centre for Diarrhoeal Disease Research, Bangladesh (ICDDR,B), GPO Box 128, Dhaka 1000, Bangladesh

${ }^{4}$ Department of Women's and Children's Health, International Maternal and Child Health, Uppsala University, Akademiska Sjukhuset, SE-751 85 Uppsala, Sweden

(Received 11 October 2010 - Revised 28 February 2011 - Accepted 7 March 2011 - First published online 1 June 2011)

\begin{abstract}
Ca status in the uterus during pregnancy has been suggested to affect fetal growth and size at birth. In Bangladesh, low Ca levels in pregnant women and low birth weight in infants are common. The present study explored the association between Ca levels in cord blood and newborn size at birth (birth weight and birth length) in Bangladesh. Samples and data included 223 women with live-born singleton deliveries in rural Bangladesh. Newborn weight and length were measured at birth. From cord blood obtained at delivery, Ca, 25-hydroxy vitamin D, bone-specific alkaline phosphatase and intact parathyroid hormone levels were determined. An association between size at birth and Ca levels in cord blood was found (birth weight, $P=0.022$; birth length, $P=0 \cdot 001$ ). Associations between Ca and newborn size were further analysed using multivariate regression analyses. After adjusting for several covariates of characteristics in mothers and newborns (gestational weeks at birth, sex of newborn, socio-economic status, maternal height, BMI, age and season at birth), birth length still exhibited a significant relationship with Ca levels in cord blood (birth length, $P=0 \cdot 030$ ). The present study indicates that Ca status in cord blood might be associated with the birth length of newborns. Ca levels during gestation may affect fetal growth.
\end{abstract}

Key words: Calcium: Size at birth: Bangladesh: Fetal growth

Low-birth weight (LBW) infants remain at much higher risk of both neonatal mortality and morbidity than infants with normal weight at birth ${ }^{(1)}$. According to a report by the WHO, LBW has been defined as weight at birth of $<2500 \mathrm{~g}^{(2)}$. This is based on epidemiological observations showing that infants weighing $<2500 \mathrm{~g}$ are approximately 20 -fold more likely to die than heavier babies ${ }^{(3)}$. There are overwhelmingly more cases of LBW in developing countries than in developed countries ${ }^{(2)}$. More than 20 million infants worldwide, representing $15.5 \%$ of all births, are born with LBW, $95.6 \%$ of them in developing countries. Half of all LBW babies are born in south-central Asia, where more than one-quarter $(27 \%)$ of all infants weigh $<2500 \mathrm{~g}$ at birth. LBW is closely associated with fetal and neonatal mortality and morbidity, inhibited growth and cognitive development, and chronic diseases later in life $\mathrm{e}^{(1,4)}$. Many factors have been reported as determinants of LBW, e.g. low socio-economic status (SES), maternal weight and nutritional status ${ }^{(5-7)}$, and they play important roles in determining the birth weight and future health of infants. Birth length has also been reported to be strongly associated with the development of the infant at 12 months ${ }^{(8)}$. Thus, newborn size has important implications for mortality, morbidity, subsequent growth and development. It is also a reflection of prenatal growth and the intra-uterine environment.

Maternal nutrition during pregnancy has a significant effect on fetal and infant growth, as does maternal health. Ca is an essential element of nutrition and is important as a primary structural constituent of the skeleton, as well as necessary for proper soft tissue functioning in muscle contraction,

Abbreviations: 25-OH VD, 25-hydroxy vitamin D; BALP, bone-specific alkaline phosphatase; BMC, bone mineral content; ICDDR,B, International Centre for Diarrhoeal Disease Research, Bangladesh; HDSS, health and demographic surveillance system; iPTH, intact parathyroid hormone; IQR, interquartile range; LBW, low-birth weight; MINIMat, Maternal and Infant Nutrition Interventions in Matlab; SES, socio-economic status.

*Corresponding author: M. Doi, fax +8129853 3489, email c0630258@md.tsukuba.ac.jp 
nerve conduction, hormone release and other physiological actions. A fetus demands Ca for growth; therefore, a pregnant woman requires more $\mathrm{Ca}$ than a non-pregnant woman ${ }^{(9)}$. The total $\mathrm{Ca}$ accretion rate of the fetus increases from approximately $50 \mathrm{mg} / \mathrm{d}$ at 20 weeks' gestation to $330 \mathrm{mg} / \mathrm{d}$ at 35 weeks' gestation. For the third trimester of pregnancy, $200 \mathrm{mg} / \mathrm{d}$ is considered the average accretion rate ${ }^{(10)}$. Ca for the fetus is transported via the placenta, and bone mineralisation in the fetus increases during pregnancy ${ }^{(11)}$. Regarding fetal growth, among infants with very LBW $(<1500 \mathrm{~g})$, their bone mineral content (BMC) and bone mass are decreased, which is related to the deficiency of a mineral, e.g. $\mathrm{Ca}^{(12)}$. Previous studies revealed that rickets is more common in infants with very LBW than in heavier infants ${ }^{(13,14)}$. Other findings have shown that infant BMC is correlated with birth weight ${ }^{(15)}$.

Several Ca supplementation trials for pregnant women have previously been conducted to determine the effects in improving maternal Ca nutritional status. A few of them reported that higher birth weights were associated with $\mathrm{Ca}$ supplementation $^{(16)}$; however, others did not find that Ca supplementation in pregnant women with low $\mathrm{Ca}$ intake influenced fetal somatic growth, skeletal growth or size at birth ${ }^{(17-19)}$. During pregnancy, Ca and vitamin D metabolism, e.g. Ca absorption and excretion, are physiologically altered ${ }^{(9,20,21)}$. In addition, seasonal influences might also need to be considered $^{(22-24)}$. Furthermore, data on the actual status of $\mathrm{Ca}$ or Ca-related biomarkers in utero that might affect bone metabolism and fetal growth, not those in mothers or newborns, are meagre.

In rural Bangladesh, the International Centre for Diarrhoeal Disease Research, Bangladesh (ICDDR,B) initiated a study called 'Maternal and Infant Nutrition Interventions in Matlab' (MINIMat), randomising all pregnant women in Matlab to receive a combination of protein, energy and micronutrient supplements and exploring combinations of pre- and postnatal nutritional interventions to address the issues of maternal, fetal and infant malnutrition. In a subcohort of infants from the main study, we aimed to investigate the association between levels of $\mathrm{Ca}$ and Ca-related biomarkers in umbilical cord blood and newborn size at birth, taking into account other variables. As vitamin D levels are known to change depending on the season, we also examined seasonal effects on the levels of these biomarkers.

\section{Experimental methods}

\section{Study area}

The study area, Matlab, is located $53 \mathrm{~km}$ southeast of the capital Dhaka. Since 1966, the ICDDR,B has organised a health and demographic surveillance system (HDSS) in the Matlab area. The HDSS covers a population of approximately 220000 . Community health research workers visit every household on a monthly basis to update information on demographic events, i.e. marriage, pregnancy, birth, death and in- and outmigration, as well as to collect information on the morbidity of children younger than 5 years of age and women of childbearing age. In 2001, the ICDDR,B initiated the MINIMat study for subsequent investigations of the effects on size at birth, gestational age at birth, fetal loss and infant mortality.

\section{Study population and design}

In the MINIMat study, through the monthly home visit by community health workers, pregnant women were identified by a history of a missed menstrual period. Upon identification of pregnancy, usually at about 6-10 weeks of gestation, women were advised to visit the respective health facility in the area for confirmation of pregnancy by ultrasound and antenatal care. If they fulfilled certain inclusion criteria ${ }^{(25)}$, the pregnant women were thereafter invited to be enrolled into the MINIMat study. Around 5000 pregnant women were recruited in the main MINIMat trial from November 2001 to October 2003. The present study was nested into the MINIMat study. For the present study, we collected cord blood from all newborns delivered at all of the Matlab subcentre clinics during daytime hours (07.00-14.30 hours) of working days (Sunday-Thursday) from April 2003 to June 2004. The term of sample collection for this nested study was the second half of the main study. Because of the logistics of the cord blood collection, we did not collect blood from newborns delivered at home or those born in the evening or at night. In Bangladesh, most pregnant women give birth at home. The proportion of subcentre clinic deliveries was $11.9 \%$ of all pregnancies at Matlab in $2004^{(26)}$.

\section{Anthropometric measurements}

Birth weight and length were measured immediately following delivery in subcentre clinics. Birth weights of all infants were measured by beam scales, which are accurate to $10 \mathrm{~g}$ (SECA, Hamburg, Germany). Locally manufactured collapsible length boards, which are precise to $1 \mathrm{~mm}$, were used to measure the recumbent length of newborn infants. The recumbent length was measured according to standard procedures $^{(27)}$. Maternal weight and height were measured at the first visit by pregnant mothers to the subcentre clinics (about 8 weeks of pregnancy).

\section{Cord blood sample}

At birth, a cord blood sample from the umbilical vein was collected into lithium heparin tubes by a trained research physician/nurse at subcentre clinics. Then, cord blood was transferred to the Matlab hospital. The Matlab hospital has a supporting laboratory for sample handling, freezer storage and sample transport to the Dhaka laboratory. From the obtained cord blood, Ca, albumin, 25-hydroxy vitamin D (25-OH VD), bone-specific alkaline phosphatase (BALP) and intact parathyroid hormone (iPTH) levels were determined in the nutrition biochemistry laboratory in Dhaka. All cord blood samples were measured for $\mathrm{Ca}$ and albumin. For 25$\mathrm{OH}$ VD, BALP and PTH, taking into account the feasibility and the available resources, 100 samples were randomly selected. The estimated sample size of 100 was based on the 
primary outcome variables of birth size, previous reports in studies using cord blood and the use of Student's $t$ test $^{(28,29)}$.

\section{Calcium and calcium-related biomarker assessment}

Cord blood was fractionated using serum separator tubes (BD Biosciences, Franklin Lakes, NJ, USA), and serum was stored at $-80^{\circ} \mathrm{C}$. Serum $\mathrm{Ca}$ and albumin were assayed using the QuantiChrom Ca assay kit (BioAssay Systems, Hayward, CA, USA). The inter-assay CV for these analyses were $8.6 \%$ of low-dose control and $5 \cdot 2 \%$ of high-dose control. Ca values were adjusted for albumin concentrations using the method described by Klemm \& Klein ${ }^{(30)}$. Serum $25-\mathrm{OH}$ VD levels were measured using the 25-hydroxyvitamin D EIA kit (Immunodiagnostic Systems Limited, Boldon, Tyne and Wear, UK). The inter-assay $\mathrm{CV}$ were $10.5 \%$ for low-dose control and $10.4 \%$ for high-dose control. Serum BALP levels were determined using the Ostase BAP kit (Immunodiagnostic Systems Limited) according to the manufacturer's recommendations. The inter-assay $\mathrm{CV}$ for these analyses were $6.8 \%$ for lowdose control and $4.8 \%$ for high-dose control. Serum iPTH levels were measured using the PTH Intact ELISA kit (DRG International, Inc., Mountainside, NJ, USA) according to the manufacturer's recommendations. The inter-assay $\mathrm{CV}$ for these analyses were $11.0 \%$ for low-dose control and $10.3 \%$ for high-dose control.

\section{Socio-economic status}

SES was estimated using a wealth index based on information about household assets and estimated by principal component analysis, producing a weighted score ${ }^{(31)}$. Scores were categorised into quintiles, with category 1 representing the poorest and category 5 the richest.

\section{Statistical analysis}

Maternal age and season at birth were categorised into three groups. Maternal age was grouped as follows: below 20 years old $(<20$ years); from 20 to 29 years old $(20-29$ years); over 30 years old ( $\geq 30$ years). Season at birth was divided into three seasons in Bangladesh: the hot and dry season (March-June); the monsoon season (July-September); winter (October-February) ${ }^{(32)}$. As an indicator of SES, a wealth index patterned in the quintile format described earlier was included in the analysis.

First, the association between size at birth, as the dependent variable, and $\mathrm{Ca}, 25-\mathrm{OH}$ VD, BALP and $\mathrm{PTH}$, as independent variables, were tested by simple linear regression. Next, a linear regression analyses were performed to select the covariates for multivariate regression analyses ${ }^{(6,7,33)}$. The covariates were maternal weight, height, BMI, category of maternal age, parity, SES evaluated by the wealth index, educational years, gestational weeks at birth, sex of newborn and season at birth. Finally, the multivariate regression analyses were performed. Categorical variables were entered in the model by producing dummy variables. Appropriate statistical methods were selected for the data being analysed.
The aforementioned analyses were conducted using the statistical package R (version 2.10.1; R Foundation for Statistical Computing, Vienna, Austria) ${ }^{(34)}$.

\section{Ethics}

The present study was conducted according to the guidelines laid down in the Declaration of Helsinki, and all procedures involving human subjects were approved by the Research Review and Ethical Review Committees, ICDDR,B, Dhaka, Bangladesh. Written informed consent was obtained from all subjects.

\section{Results}

\section{Characteristics of mothers and newborns}

A total of 225 cord blood samples were collected. The background characteristics of 225 mothers and newborns are

Table 1. Characteristics of mothers, newborns, calcium and calcium-related biomarkers in cord blood

(Mean values, standard deviations, medians, interquartile ranges (IQR), number of participants and percentages)

\begin{tabular}{|c|c|c|}
\hline Variables & Mean & SD \\
\hline \multicolumn{3}{|l|}{ Mothers ( $n$ 225) } \\
\hline Age (years) & $25 \cdot 8$ & 5.9 \\
\hline Maternal weight $(\mathrm{kg})$ & $46 \cdot 4$ & $8 \cdot 1$ \\
\hline Maternal height $(\mathrm{cm})$ & $150 \cdot 7$ & $5 \cdot 5$ \\
\hline Maternal BMI $\left(\mathrm{kg} / \mathrm{m}^{2}\right)$ & $20 \cdot 4$ & 2.9 \\
\hline Parity & $1 \cdot 2$ & 1.4 \\
\hline \multicolumn{3}{|l|}{ Educational years } \\
\hline$<7$ years $(\%)$ & \multicolumn{2}{|c|}{$45 \cdot 3$} \\
\hline \multicolumn{3}{|l|}{ Newborns (n 225) } \\
\hline Birth weight $(\mathrm{g})$ & 2812 & 386 \\
\hline Birth length $(\mathrm{cm})$ & $48 \cdot 1$ & 1.9 \\
\hline \multicolumn{3}{|l|}{ Sex of newborn infant } \\
\hline Male (\%) & $49 \cdot 8$ & \\
\hline \multicolumn{3}{|l|}{ Pre-term birth } \\
\hline < 37 weeks (\%) & \multicolumn{2}{|c|}{$7 \cdot 1$} \\
\hline \multicolumn{3}{|l|}{ Biochemical markers } \\
\hline \multicolumn{3}{|l|}{ Serum Ca $\left(\mathrm{mg} / \mathrm{l}^{\star} \dagger\right.$} \\
\hline Median & \multirow{2}{*}{\multicolumn{2}{|c|}{$\begin{array}{c}34 \\
22-63\end{array}$}} \\
\hline IQR & & \\
\hline$n$ & \multicolumn{2}{|c|}{223} \\
\hline \multicolumn{3}{|c|}{ Serum $25-\mathrm{OH}$ VD $(\mathrm{ng} / \mathrm{ml}) \dagger \ddagger$} \\
\hline Median & \multirow{2}{*}{\multicolumn{2}{|c|}{$\begin{array}{c}23 \cdot 8 \\
18 \cdot 6-30.8\end{array}$}} \\
\hline IQR & & \\
\hline$n$ & \multicolumn{2}{|c|}{98} \\
\hline \multicolumn{3}{|l|}{ Serum BALP $(\mu \mathrm{g} / \mathrm{l}) \dagger \ddagger$} \\
\hline Median & \multirow{2}{*}{\multicolumn{2}{|c|}{$\begin{array}{c}16 \cdot 3 \\
13 \cdot 3-19 \cdot 1\end{array}$}} \\
\hline IQR & & \\
\hline$n$ & \multicolumn{2}{|c|}{99} \\
\hline \multicolumn{3}{|l|}{ 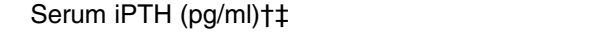 } \\
\hline Median & \multirow{2}{*}{\multicolumn{2}{|c|}{$0.0-5.3$}} \\
\hline IQR & & \\
\hline$n$ & \multicolumn{2}{|c|}{99} \\
\hline
\end{tabular}

25-OH VD, 25-hydroxy vitamin D; BALP, bone-specific alkaline phosphatase; iPTH, intact parathyroid hormone.

* Ca was adjusted for albumin.

† Two participants were excluded as outliers for further analysis.

¥ The 100 samples were randomly selected for Ca-related biomarkers. One or two samples were excluded as outliers. 
presented in Table 1. The age of mothers ranged from 14 to 43 years with a mean age of 25.8 (SD 5.9) years. The mean weight and height of the women at about week 8 of gestation were $46 \cdot 4$ (SD $8 \cdot 1$, range $25 \cdot 0-76 \cdot 4) \mathrm{kg}$ and $150 \cdot 6$ (SD 5.5, range $134 \cdot 5-167 \cdot 0) \mathrm{cm}$, respectively. Approximately one-third $(28.9 \%)$ of the women were malnourished (BMI $\left.<18.5 \mathrm{~kg} / \mathrm{m}^{2}\right)$; two-fifths $(40.0 \%)$ of the women were primigravidas. Approximately half (45.3\%) of the women had completed less than seven educational years. The mean size at birth (birth weight and length) was 2812 (SD 389, range $1820-4000) \mathrm{g}$ and $48 \cdot 1$ (SD $1 \cdot 9$, range $43 \cdot 0-58 \cdot 1) \mathrm{cm}$, respectively. Approximately twenty (19.6)\% of the newborns had LBW. The mean gestational age at birth was $39 \cdot 2$ (SD 1.5) weeks. Approximately $7 \%$ of the infants were born pre-term $(<37$ weeks). Outliers were identified by the univariate analysis. The two outliers were excluded for further analysis because of birth length $>58 \mathrm{~cm}$ and iPTH levels $>133 \mathrm{pg} / \mathrm{ml}$. Serum Ca levels were determined in 223 samples.

\section{Calcium and calcium-related biomarkers in cord blood}

The levels of $\mathrm{Ca}$ in cord blood are summarised in Table 1. $\mathrm{Ca}, 25-\mathrm{OH}$ VD, BALP and iPTH were not normally distributed, even after log transformation. Therefore, non-parametric tests were used for those variables. The median value of $\mathrm{Ca}$ was 34 (interquartile range (IQR) $22-63 \mathrm{mg} / 1 ; n$ 223). For Ca-related biomarkers, one or two samples were excluded as the aforementioned outliers. The median scores and IQR for the Ca-related biomarkers were as follows: $25-\mathrm{OH}$ VD $(23.8 \mathrm{ng} / \mathrm{ml} ; \quad$ IQR $18.6-30.8 ; n$ 98); BALP $(16.3 \mu \mathrm{g} / \mathrm{l}$; IQR $13 \cdot 3-19 \cdot 1 ; n$ 99); iPTH $(0.5 \mathrm{pg} / \mathrm{ml} ;$ IQR $0 \cdot 0-5 \cdot 3 ; n$ 99). Serum $\mathrm{Ca}, 25-\mathrm{OH}$ VD, BALP and iPTH levels were not associated with allocation to food and micronutrient randomisation groups in the MINIMat study. Ca was not contained in the micronutrient tablets used in the MINIMat study.

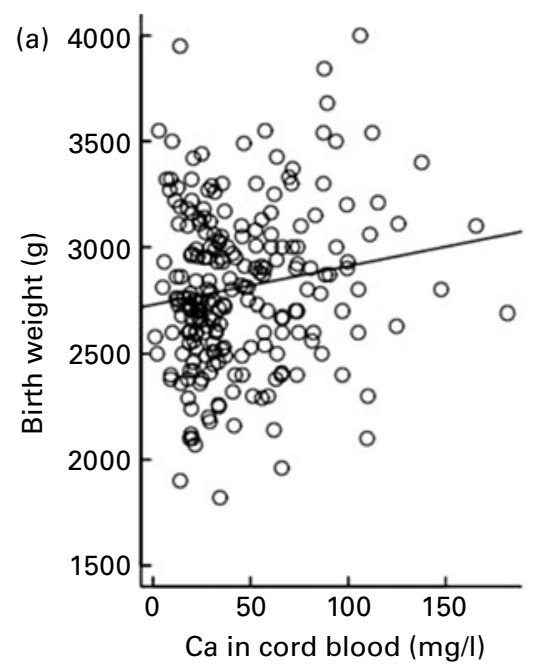

Relationship among size at birth, calcium and calcium-related biomarkers

The associations among size at birth, Ca and Ca-related biomarkers were assessed by a simple linear regression analysis. The associations between $\mathrm{Ca}$ and size at birth were examined by scatter plots that included a linear regression line (Fig. 1). The regression analysis showed a significant association between $\mathrm{Ca}$ levels and size at birth (birth weight: $\beta 1.83$, 95\% CI 0.27, 3.39; birth length: $\beta$ 0.012, 95\% CI 0.005, 0.020). Serum 25-OH VD, BALP and iPTH levels were not significantly related to birth weight and length. For the relationship between $\mathrm{Ca}$ and size at birth confirmed by simple linear regression, a further multivariate analysis was conducted.

\section{Simple linear regression analysis of size at birth, calcium and selected covariates}

Table 2 shows the simple regression analysis of $\mathrm{Ca}$, size at birth and selected covariates for multivariate regression analysis. For birth weight, maternal height $(P<0 \cdot 001)$, maternal BMI $(P=0.002)$, categories of maternal age $(P=0.032)$, wealth index $(P=0.046)$ and gestational weeks at birth $(P<0.001)$ were found to be correlated significantly. For birth length, maternal height $(P<0.001)$, maternal BMI $(P=0.025)$, gestational weeks at birth $(P<0.001)$ and the sex of newborns $(P=0.013)$ were significantly associated. Ca levels in cord blood were significantly related to the sex of newborns $(P=0 \cdot 011)$. Ca levels in cord blood were significantly different among seasons at birth $(P<0 \cdot 001)$.

Among covariates, maternal weight was associated with maternal height $(r 0.55, P<0.001 ; n$ 223) and maternal BMI ( $r$ 0.91, $P<0.001 ; n$ 223). Parity was significantly different in the category of maternal age $(P<0 \cdot 001 ; n 223)$. The mother's educational years and wealth index were positively correlated ( $r$ 0.44, $P<0.001 ; n$ 223). Considering correlations between these covariates, maternal weight, parity and mother's educational years were excluded.

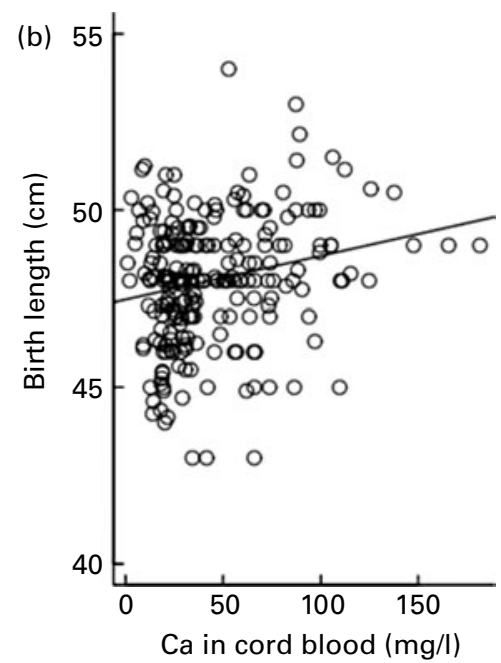

Fig. 1. Scatter plot and estimated simple linear regression equation for the association between calcium in cord blood and newborn size. (a) Birth weight $(Y=2728+1.83 X ; n 223 ; P=0.022)$ and $(b)$ birth length $(Y=47.5+0.012 X ; n 223 ; P=0.001)$. 
Table 2. Simple regression analysis with calcium, size at birth and selected covariates ( $\beta$-Coefficients and standard errors)

\begin{tabular}{|c|c|c|c|c|c|c|c|c|c|}
\hline & \multicolumn{3}{|c|}{ Birth weight ( $n$ 223) } & \multicolumn{3}{|c|}{ Birth length ( $n$ 223) } & \multicolumn{3}{|c|}{$\mathrm{Ca}(n$ 223) } \\
\hline & $\beta$ & SE & $P$ & $\beta$ & SE & $P$ & $\beta$ & SE & $P$ \\
\hline Maternal height $(\mathrm{cm})$ & $21 \cdot 2$ & 4.4 & $<0.001^{*}$ & 0.09 & 0.02 & $<0.001^{*}$ & -0.01 & 0.04 & 0.893 \\
\hline Maternal BMI $\left(\mathrm{kg} / \mathrm{m}^{2}\right)$ & $26 \cdot 8$ & 8.7 & $0.002^{*}$ & 0.10 & 0.04 & $0.025^{\star}$ & 0.05 & 0.07 & 0.519 \\
\hline Category of maternal age (years) & $89 \cdot 2$ & $41 \cdot 2$ & $0.032^{*}$ & 0.25 & 0.20 & 0.204 & -0.12 & 0.35 & 0.731 \\
\hline Wealth index (quintile) & $37 \cdot 0$ & 18.4 & $0.046^{\star}$ & 0.14 & 0.09 & 0.117 & 0.17 & 0.16 & 0.286 \\
\hline Gestational weeks at birth & 117.9 & 14.7 & $<0.001^{*}$ & 0.49 & 0.07 & $<0.001^{*}$ & 0.19 & 0.14 & 0.183 \\
\hline Sex of newbornt & -95.4 & 50.6 & 0.061 & -0.61 & 0.24 & $0.013^{*}$ & -1.09 & 0.42 & $0.011^{*}$ \\
\hline
\end{tabular}

${ }^{*} P$ values were significantly different for birth weight, birth length and $\mathrm{Ca}$ by covariates $(P<0.05)$.

$\dagger$ Sex of newborn, females $v$. males.

In the present study, three adjusted models were created for birth weight and birth length. Model I was controlled with the gestational weeks at birth, sex of the newborn and wealth index. For model II, covariates on maternal characteristics - maternal height, BMI and category of maternal age were added to model I. Finally, season at birth was included in the fully adjusted multivariate model (model III).

\section{Multivariate regression analysis between size at birth and calcium}

Table 3 shows the multivariate regression analysis of size at birth (birth weight and length) and Ca in cord blood. Regarding birth weight, Ca was not correlated significantly after adjustment for gestational weeks at birth, sex of the newborn and wealth index (model I, $P=0 \cdot 178$ ). Additional adjustment for covariates on maternal characteristics (model II) did not reveal an association between $\mathrm{Ca}$ levels and birth weight ( $P=0 \cdot 138$ ). After adjusting for season at birth (model III), no association was observed between $\mathrm{Ca}$ levels and birth weight $(P=0 \cdot 132)$.

We also examined the associations of $\mathrm{Ca}$ and birth length in three types of adjusted models. Ca levels were significantly associated with birth length in the multivariate model I $(P=0 \cdot 019)$. We examined the associations of $\mathrm{Ca}$ and size at

Table 3. Multivariate regression analysis of size at birth and calcium in cord blood

( $\beta$-Coefficients and standard errors)

\begin{tabular}{|c|c|c|c|c|c|c|}
\hline & \multicolumn{3}{|c|}{$\begin{array}{l}\text { With gestational } \\
\text { week at birth }\end{array}$} & \multicolumn{3}{|c|}{$\begin{array}{l}\text { Without gestational } \\
\text { week at birth }\end{array}$} \\
\hline & $\beta$ & SE & $P$ & $\beta$ & SE & $P$ \\
\hline \multicolumn{7}{|c|}{ Birth weight ( $n$ 223) } \\
\hline Model I* & 0.95 & 0.70 & 0.178 & $1 \cdot 61$ & 0.79 & 0.042 \\
\hline Model II† & 1.02 & 0.68 & 0.138 & 1.58 & 0.76 & 0.037 \\
\hline Model III & 1.28 & 0.85 & 0.132 & $2 \cdot 04$ & 0.94 & 0.031 \\
\hline \multicolumn{7}{|c|}{ Birth length ( $n$ 223) } \\
\hline Model I* & 0.008 & 0.004 & 0.019 & 0.011 & 0.004 & 0.004 \\
\hline Model II† & 0.009 & 0.003 & 0.014 & 0.011 & 0.004 & 0.003 \\
\hline Model III‡ & 0.009 & 0.004 & 0.030 & 0.013 & 0.005 & 0.007 \\
\hline
\end{tabular}

* Adjusted for gestational weeks at birth, sex of newborn and wealth index in quintile.

† Additional adjustment for maternal height, maternal BMI and category of maternal age to model I.

‡ Simultaneously adjusted for season at birth to model III. birth controlled for the aforementioned three covariates and covariates on maternal characteristics (model II) and confirmed a significant result $(P=0 \cdot 014)$. Even after adjusting for season at birth, Ca levels remained significantly associated with only birth length in the fully adjusted model III $(P=0.030)$. Normal quantile-quantile plots were made to examine the residuals of the multivariate models. As a result, the residuals were normally distributed, and no extreme outlier existed.

Considering that the gestational weeks at birth would be an intermediate factor, the aforementioned three multivariate analyses were also conducted without the gestational weeks at birth (Table 3). The multivariate regression analysis without the gestational age at birth revealed the significant relationships between size at birth and $\mathrm{Ca}$ in the three models (birth weight, model I: $P=0.042$; model II: $P=0.037$; model III: $P=0.031$; birth length, model I: $P=0.004$; model II: $P=0.003$; model III: $P=0 \cdot 007)$.

\section{Seasonal change in calcium and calcium-related biomarkers}

Table 4 shows the characteristics of $\mathrm{Ca}$ and Ca-related biomarkers in cord blood depending on the season at birth. By using the Steel-Dwass test for multiple tests, the Ca levels in cord blood were significantly different among the three seasons. Compared with the hot and dry season, the 25-OH VD concentration in cord blood during the winter and monsoon seasons was significantly lower (Table 4). Serum 25-OH VD in cord blood was negatively correlated with Ca $(\rho=-0 \cdot 21$, $P=0.04 ; n$ 98). The mean BALP and iPTH concentrations in cord blood were not significantly different among the three seasons.

\section{Discussion}

The present study found an association between the level of $\mathrm{Ca}$ in umbilical cord blood and infant size at birth in rural Bangladesh. In particular, the positive association of $\mathrm{Ca}$ levels with birth length remained after adjustment for covariates. The present study is the first to clarify the relationship between Ca levels at birth and newborn size after adjustment for other covariates. Participants of this cohort were recruited over an entire year so that seasonal variations in independent 
and dependent variables could be considered. In the present study, the values of Ca-related biomarkers in cord blood in rural Bangladesh were determined for the first time. Those could be considered as reference values for future studies.

Whether Ca levels in cord blood are associated with birth length are heretofore unknown. In the present study, Ca levels in cord blood had an association with birth length both by simple linear regression and by multivariate regression analysis. Birth length was also reported to be as important a factor as birth weight for infants. Morris et $a l^{(8)}$ reported that birth length was strongly associated with child development at 12 months as measured by the Denver II developmental screening test. In addition, infants who were born both short and with a low ponderal index were at increased risk of mortality and severe morbidity in infancy. Ca levels in cord blood were found to be significantly associated with birth length in the present study. These data might suggest that low Ca levels in pregnant women may negatively affect fetal bone development by limiting the amount of $\mathrm{Ca}$ provided to the fetus, and this would lead to a lower birth length. Chang et al. ${ }^{(35)}$ reported that a significant association between maternal dairy intake and fetal femur growth was found among pregnant African-American adolescents. In their study, dairy intake had a significant positive effect on fetal femur growth after adjustment for gestational age, biparietal diameter, maternal age and height, and BMI. Fetal femur length was significantly lower in the lowest dairy-intake group than in the highest dairy-intake group. Even though it is not clear whether the differences in femur length during pregnancy corresponded to differences in birth length, Ca levels in pregnancy might be related to birth length in addition to bone length and fetal length. To increase newborn birth length, pregnant mothers might be recommended to consume more foods containing $\mathrm{Ca}$.

Previous reports have shown that the Ca level of cord blood is related to birth weight ${ }^{(28,29)}$. Bogden et al $^{(28)}$ found that $\mathrm{Ca}$ levels in cord blood were significantly lower in LBW babies than in control babies weighing more than $2500 \mathrm{~g}$. Their result is consistent with that of Elizabeth et al. who also found lower Ca levels in LBW infants. Elizabeth et al. ${ }^{(29)}$ classified newborns as pre-term LBW, term LBW and term controls, and confirmed that pre-term and term LBW babies were born with significantly lower Ca levels in cord blood than term control babies. These studies have revealed that $\mathrm{Ca}$ levels in cord blood were associated with birth weight by simple bivariate analysis without adjustment for other predictors. In the present study, the association of Ca levels in cord blood with birth weight was demonstrated by simple linear regression. $\mathrm{Ca}$ is a primary component of bone, and BMC is related to birth weight and infant weight ${ }^{(36,37)}$. Ca might lead to a higher birth weight via increasing $\mathrm{Ca}$ transfer to the fetus and increasing the BMC. LBW is closely associated with fetal and neonatal mortality and morbidity, inhibited growth and cognitive development, and chronic diseases later in life ${ }^{(2)}$. The prevention of LBW is beneficial for the life of the infant. Purwar et al $^{(16)}$ conducted Ca supplementation for pregnant women to determine the effects of maternal $\mathrm{Ca}$ nutritional status on fetal growth. Their results indicated that larger 
birth weights are associated with supplementation. However, in their study, larger birth weights were not explained by $\mathrm{Ca}$ levels in cord blood.

Several predictors concerning birth weight have already been reported in previous studies, e.g. SES, maternal nutrition, maternal age, height, BMI and parity ${ }^{(6,7,33)}$. Taking into account previously reported predictors, the association between Ca levels and birth weight was not confirmed by the multivariate analysis. Birth weight, especially LBW, was known to be correlated with gestational age at birth. In the present study, the effect of gestational age on size at birth was obvious, and in particular, birth weight was more affected by gestational age than birth length. Ca has also been reported to reduce the risk of birth weight by prolonging gestation ${ }^{(38)}$. Compared with birth length, birth weight would be more explained by gestational age, and the direct effects of $\mathrm{Ca}$ on birth weight might be weaker than that on birth length. As a result, the association after adjustment could not be detected in this population. Otherwise, there might be a time lag until the change in the Ca level is reflected in the birth weight. Body length reflects skeletal growth, whereas birth weight may reflect more factors, e.g. body fat and muscle mass.

Previously, Ca levels have been reported to be positively correlated with gestational age ${ }^{(39)}$. Ca supplementation has been reported to prevent pre-term delivery and preeclampsia $^{(18,40)}$. It has been suggested that the influence of extracellular Ca concentrations might reduce smooth muscle cell tone and increase its relaxation ${ }^{(40)}$. The loosening of smooth muscle cells might prevent the pre-eclampsia and hypertension or pre-term delivery of the uterus, thereby increasing the number of gestational weeks. As a different viewpoint of this regard, Ca transport from the mother to the fetus increases skeletal growth from the second trimester to the third trimester of pregnancy ${ }^{(41)}$. With shorter gestational time, there might be still lower $\mathrm{Ca}$ amounts to be transferred through the placenta. Consequently, $\mathrm{Ca}$ in cord blood might be lower at early gestational age. If the gestational age might mediate the association between $\mathrm{Ca}$ and newborn size, the relationship between $\mathrm{Ca}$ and newborn size in the present multivariate analysis might be underestimated by the control of the gestational age at birth. In the present study, Ca was not significantly related to gestational age. Because of the low proportion of pre-term delivery in the present study, the relationship between $\mathrm{Ca}$ and gestational age might not be observed.

For maintaining adequate Ca levels in utero, Ca supplementation for low Ca intake might be useful. Several studies of Ca and/or vitamin D supplementation for pregnant women have already been conducted, of which many studies have indicated a reduced risk of pregnancy-induced hypertension and pre-eclampsia $^{(16,17)}$. Ca levels in cord blood have already been reported to be correlated with maternal Ca status ${ }^{(42)}$. It appears that increasing Ca levels in pregnancy with those supplements will lead to an increase in Ca in utero, and that the positive effects of Ca supplementation on fetal growth or newborn size will result. In a systematic review, the overall results of trials of Ca supplementation demonstrated a significant $17 \%$ reduction in the risk of $\mathrm{LBW}^{(38)}$. However, some trials have found that Ca supplementation in pregnant women with low Ca intake does not appear to have an impact on birth weight and length ${ }^{(17-19,40,43)}$. From the present results, if the appropriate amount of $\mathrm{Ca}$ is transported in utero and is kept at appropriate levels, size at birth might be significantly improved. During pregnancy, physiological mechanisms involved in $\mathrm{Ca}$ homeostasis are also known to be altered because $\mathrm{Ca}$ metabolism, e.g. Ca absorption, renal $\mathrm{Ca}$ excretion, Ca resorption, Ca transportation via the placenta and increases in bone turnover in pregnancy and lactation, is working complexly for the fetus and the mother ${ }^{(10,20,44)}$. Taking this into account, a method to maintain appropriate Ca levels might be important. We also need to consider the amount of Ca supplementation with respect to other factors, e.g. geography, nutritional status or cultural habits. Bangladeshi women have been reported to usually have low Ca intake status $^{(45)}$, and there is also a high prevalence of rickets in a few areas in Bangladesh ${ }^{(46)}$, although none of these areas was included in the present study field.

Other interesting findings of the present study were the significantly higher values of 25-OH VD levels in the hot and dry season (March-June) and the differences in the Ca levels among the three seasons. In the present study, even though the season at birth did not affect birth weight and length, a seasonal difference in 25-OH VD/Ca levels was confirmed. In Bangladesh, lower amounts of UV are radiated in the monsoon season, which means that synthesis and 25-OH VD stock might decrease in that season, and that Ca levels subsequently decline. The maternal level of 25-OH VD changes seasonally. $25-\mathrm{OH} \mathrm{VD}$ is transported from the mother via the placenta to the fetus ${ }^{(47)}$. Seasonal differences in maternal 25-OH VD levels might give rise to the different maternal $\mathrm{Ca}$ levels. $\mathrm{Ca}$ is also transported from the mother via the placenta to the fetus, and transported $\mathrm{Ca}$ is correlated with the maternal $\mathrm{Ca}$ level $^{(42)}$. Consequently, the Ca levels in cord blood might be changed simultaneously with the maternal Ca level. There would be a time interval between the change in the $25-\mathrm{OH}$ VD level and that in the Ca level because of the pathways of metabolism and transport. Unfortunately, maternal levels of 25-OH VD and Ca during pregnancy or maternal BMC as the indicator of total $\mathrm{Ca}$ status were not measured in the present study. Earlier studies have revealed seasonal variations in serum 25-OH VD concentrations in both pregnant women and their newborn infants, with low values in winter ${ }^{(22,48)}$. Namgung et al. found significant seasonal differences; summer-born infants had significantly lower BMC, higher 1,25-vitamin D levels and lower total Ca levels than winterborn infants. A positive relationship has also been reported between $\mathrm{BMC}$ and birth weight ${ }^{(15)}$. According to the present results, the protective effects of vitamin D supplementation would be more expected for pregnant women during the winter and monsoon seasons in Bangladesh.

The present study reported Ca-related biomarker values of cord blood in Bangladesh. Even though newborns with LBW usually tend to have low Ca levels ${ }^{(28)}$ and $20 \%$ of newborns were LBW in the present study, Ca levels were lower than the usually reported value of $90 \mathrm{mg} / \mathrm{l}^{(49,50)}$. Poor Ca intake by Bangladeshi women has been reported. Islam et al. ${ }^{(45)}$ 
reported that more than half of the women in the low-SES group had Ca intake below $200 \mathrm{mg} / \mathrm{d}$. In their study, $95 \%$ of women in the low-SES group and $47 \%$ of those in the highSES group had a daily Ca intake on average of $<400 \mathrm{mg} / \mathrm{d}$. Low Ca intake might influence low Ca levels in cord blood. Among different ethnicities, the metabolism of $\mathrm{Ca}$ and $\mathrm{Ca}$ related biomarkers has been reported to be different ${ }^{(51)}$. In addition, it is known that the season, meteorology or environmental toxic materials might affect the Ca levels ${ }^{(50,52)}$.

The mean values of $25-\mathrm{OH} \mathrm{VD}$ and $\mathrm{iPTH}$ were similar to previously reported data ${ }^{(50,53)}$. Concerning $25-\mathrm{OH}$ VD, BALP and $\mathrm{iPTH}$, there were no associations with newborn size in the present study. Nonetheless, there have been previous reports indicating the relationships between size at birth and Ca-related biomarkers. Leffelaar et al. ${ }^{(54)}$ indicated that infants from women with deficient vitamin D levels had lower birth weights than those from women with adequate vitamin $\mathrm{D}$ levels in a large multiethnic cohort. In the vitamin D supplementation trials in the Cochrane database, mothers had a lower number of LBW infants ${ }^{(55)}$. Morely et al. ${ }^{(56)}$ reported that the maternal parathyroid hormone concentration at 28-32 weeks was positively related to knee-heel length, birth weight, and mid-upper arm and calf circumferences of infants at birth independently. Regarding the relationship between bone growth and Ca-related biomarkers, previous studies suggested that maternal vitamin D status affects bone mineral accrual during the intra-uterine period and influences bone size ${ }^{(56,57)}$. These findings suggest that Ca-related nutritional factors have an aetiological function for newborn size.

The strength of the present study is that we could utilise cord blood. By using cord blood, we were able to evaluate the actual $\mathrm{Ca}$ status of the newborn at birth and to analyse the association of $\mathrm{Ca}$ with size at birth. Increased $\mathrm{Ca}$ levels in utero were clarified to be directly associated with birth length. Moreover, samples were obtained from the large population under the HDSS. By utilising the HDSS, characteristics of the mother and household were obtained appropriately, and following the participants in the future will be possible.

The present study had some limitations. First, the sample size was small. Blood samples were taken from only a minority of the main study subjects. Caution should be taken in applying the present results to all newborns, and further confirmation is required. Next, we did not consider the food intake of the mothers during pregnancy. The effects of food intake and $\mathrm{Ca}$ status of pregnant women were not considered in the present analysis.

The present study demonstrated a significant association of Ca levels in cord blood with birth size among Bangladeshi newborns. In particular, the relationship between Ca levels in cord blood and birth length was not altered by the adjustment for gestational age at birth, maternal factor and SES. The present study also found that Ca levels in cord blood were low in Bangladesh, where the prevalence of maternal malnutrition is high. Ca may play an important role in fetal growth in this population. Further studies will be required to confirm the findings in other areas.

\section{Acknowledgements}

The MINIMat study was funded by the ICDDR,B; United Nations Children's Fund; the Swedish International Development Cooperation Agency; the United Kingdom Medical Research Council; the Swedish Research Council, Department for International Development; Child Health and Nutrition Research Initiative; Uppsala University; the US Agency for International Development; and a Grant-in-Aid for Scientific Research of the Japan Society for the Promotion of Science (no. 18256005). M. D. contributed to the design of the study, data analysis and writing of the manuscript. R. S. R. conducted the data collection, editing of the data and laboratory assessment. S. A. performed the data collection and laboratory assessment. M. O. interpreted the data. A. K. R. performed the biomarker assessment. S. E. A. contributed to the overall design and implementation of the MINIMat study. E.-C. E. provided expertise on micronutrient analysis and scientific comments to improve the manuscript. R. R. contributed to the design and implementation of the present study in Bangladesh, interpretation of the data and writing of the manuscript. Y. W. assisted in designing and implementing the study, interpreting the data and writing of the manuscript. All authors read and approved the final version of the manuscript. None of the authors has a personal or financial conflict of interest to declare. We thank the staff of the MINIMat study. We are grateful to Ms Marianne Kimura for editing the manuscript. Drs Hideto Takahashi and Embo Ma are acknowledged for their valuable comments to improve the manuscript.

\section{References}

1. McIntire DD, Bloom SL, Casey BM, et al. (1999) Birth weight in relation to morbidity and mortality among newborn infants. N Engl J Med 340, 1234-1238.

2. Wardlaw T, Blanc A, Zupan J, et al. (2004) Low Birthweight: Country, Regional and Global Estimates. Geneva/New York: WHO/UNICEF.

3. Kramer MS (1987) Determinants of low birth weight: methodological assessment and meta-analysis. Bull World Health Organ 65, 663-737.

4. Eriksson JG, Forsén T, Tuomilehto J, et al. (1999) Catch-up growth in childhood and death from coronary heart disease: longitudinal study. BMJ 318, 427-431.

5. Torres-Arreola LP, Constantino-Casas P, Flores-Hernández S, et al. (2005) Socioeconomic factors and low birth weight in Mexico. BMC Public Health 5, 20.

6. Neel NR \& Alvarez JO (1991) Maternal risk factors for low birth weight and intrauterine growth retardation in a Guatemalan population. Bull Pan Am Health Organ 25, 152-165.

7. Lone FW, Qureshi RN \& Emanuel F (2004) Maternal anaemia and its impact on perinatal outcome. Trop Med Int Health $\mathbf{9}$, 486-490.

8. Morris SS, Victora CG, Barros FC, et al. (1998) Length and ponderal index at birth: associations with mortality, hospitalizations, development and post-natal growth in brazilian infants. Int J Epidemiol 27, 242-247.

9. Kovacs CS (2008) Vitamin D in pregnancy and lactation: maternal, fetal, and neonatal outcomes from human and animal studies. Am J Clin Nutr 88, 520S-528S.

10. Prentice A (2000) Calcium in pregnancy and lactation. Annu Rev Nutr 20, 249-272. 
11. Prentice A (2003) Micronutrients and the bone mineral content of the mother, fetus and newborn. J Nutr $\mathbf{1 3 3}$, 1693S-1699S.

12. Abrams SA \& Hawthorne KM (2006) Infancy and childhood. In Calcium in Human Health, pp. 269-279 [CM Weaver and RP Heaney, editors]. Totowa, NJ: Humana Press.

13. Callenbach JC, Sheehan MB, Abramson SJ, et al. (1981) Etiologic factors in rickets of very low-birth-weight infants. J Pediatr 98, 800-805.

14. Lindroth M, Westgren U \& Laurin S (1986) Rickets in very low birthweight infants. Influence of supplementation with vitamin D, phosphorus and calcium. Acta Paediatr Scand 75, 927-931

15. Namgung R, Tsang RC, Specker BL, et al. (1993) Reduced serum osteocalcin and 1,25-dihydroxyvitamin D concentrations and low bone mineral content in small for gestational age infants: evidence of decreased bone formation rates. J Pediatr 122, 269-275.

16. Purwar M, Kulkarni H, Motghare V, et al. (1996) Calcium supplementation and prevention of pregnancy induced hypertension. J Obstet Gynaecol Res 22, 425-430.

17. Sanchez-Ramos L, Briones DK, Kaunitz AM, et al. (1994) Prevention of pregnancy-induced hypertension by calcium supplementation in angiotensin II-sensitive patients. Obstet Gynecol 84, 349-353.

18. López-Jaramillo P, Delgado F, Jácome P, et al. (1997) Calcium supplementation and the risk of preeclampsia in ecuadorian pregnant teenagers. Obstet Gynecol 90, 162-167.

19. Abalos E, Merialdi M, Wojdyla D, et al. (2010) Effects of calcium supplementation on fetal growth in mothers with deficient calcium intake: a randomised controlled trial Paediatr Perinat Epidemiol 24, 53-62.

20. Kovacs CS \& Kronenberg HM (1997) Maternal-fetal calcium and bone metabolism during pregnancy, puerperium, and lactation. Endocr Rev 18, 832-872.

21. Guéguen L \& Pointillart A (2000) The bioavailability of dietary calcium. J Am Coll Nutr 19, 119S-136S.

22. Verity CM, Burman D, Beadle PC, et al. (1981) Seasonal changes in perinatal vitamin $\mathrm{D}$ metabolism: maternal and cord blood biochemistry in normal pregnancies. Arch Dis Child 56, 943-948.

23. Zittermann A, Dembinski J \& Stehle P (2004) Low vitamin D status is associated with low cord blood levels of the immunosuppressive cytokine interleukin-10. Pediatr Allergy Immunol 15, 242-246.

24. Walters BNJ (1989) Calcium metabolism in pregnancy. Fetal Matern Med Rev 1, 213-222.

25. Rahman A, Vahter M, Smith AH, et al. (2009) Arsenic exposure during pregnancy and size at birth: a prospective cohort study in Bangladesh. Am J Epidemiol 169, 304-312.

26. International Center of Diarrhoeal Disease Research, Bangladesh - ICDDR,B (2006) Health and demographic surveillance system-Matlab: vol. 37, Registration of Health and Demographic Events 2004. Dhaka: ICDDR,B.

27. World Health Organization (1999) Interventions for Physical Growth and Psychological Development. Geneva: WHO.

28. Bogden JD, Thind IS, Louria DB, et al. (1978) Maternal and cord blood metal concentrations and low birth weight - a case-control study. Am J Clin Nutr 31, 1181-1187.

29. Elizabeth KE, Krishnan V \& Vijayakumar T (2008) Umbilical cord blood nutrients in low birth weight babies in relation to birth weight and gestational age. Indian J Med Res $\mathbf{1 2 8}$, $128-133$

30. Klemm KM \& Klein MJ (2007) Biochemical markers of bone metabolism. In Henry's Clinical Diagnosis and Management by Laboratory Methods, pp. 170-184
[RA McPherson and MR Pincus, editors]. Philadelphia: Saunders Elsevier.

31. Gwatkin DR, Rustein S, Johnson K, et al. (2000) Socioeconomic Differences in Health, Nutrition, and Population in Bangladesh. http://siteresources.worldbank.org/INTPAH/ Resources/IndicatorsOverview.pdf (accessed September 2010).

32. Moore S, Prentice A, Wagatsuma Y, et al. (2009) Early-life nutritional and environmental determinants of thymic size in infants born in rural Bangladesh. Acta Paediatr 98, $1168-1175$.

33. Deshmukh JS, Motghare DD, Zodpey SP, et al. (1998) Low birth weight and associated maternal factors in an urban area. Indian Pediatr 35, 33-36.

34. R Development Core Team (2008) R: A Language and Environment for Statistical Computing Vienna: R Foundation for Statistical Computing. http://www.R-project.org

35. Chang SC, O'Brien KO, Nathanson MS, et al. (2003) Fetal femur length is influenced by maternal dairy intake in pregnant African American adolescents. Am J Clin Nutr 77, $1248-1254$

36. Avila-Díaz M, Flores-Huerta S, Martínez-Muñiz I, et al. (2001) Increments in whole body bone mineral content associated with weight and length in pre-term and fullterm infants during the first 6 months of life. Arch Med Res 32, 288-292.

37. Dennison EM, Syddall HE, Sayer AA, et al. (2005) Birth weight and weight at 1 year are independent determinants of bone mass in the seventh decade: the Hertfordshire cohort study. Pediatr Res 57, 582-586.

38. Merialdi M, Carroli G, Villar J, et al. (2003) Nutritional interventions during pregnancy for the prevention or treatment of impaired fetal growth: an overview of randomized controlled trials. J Nutr 133, 1626S-1631S

39. Nelson N, Finnström O \& Larsson L (1989) Plasma ionized calcium, phosphate and magnesium in preterm and small for gestational age infants. Acta Paediatr Scand 78, $351-357$

40. Villar J \& Repke JT (1990) Calcium supplementation during pregnancy may reduce preterm delivery in high-risk populations. Am J Obstet Gynecol 163, 1124-1131.

41. Prentice A, Laskey MA \& Jarjou LMA (1999) Lactation and bone development: implications for the calcium requirements of infants and lactating mothers. In Nutrition and Bone Development, pp. 127-145 [JP Bonjour and RC Tsang, editors]. Lippincott-Raven/Philadelphia, Vevey, Switzerland: Nestlé Nutrition Services.

42. Schauberger CW \& Pitkin RM (1979) Maternal-perinatal calcium relationships. Obstet Gynecol 53, 74-76.

43. Belizán JM, Villar J, Gonzalez L, et al. (1991) Calcium supplementation to prevent hypertensive disorders of pregnancy. $N$ Engl J Med 325, 1399-1405.

44. Kovacs CS (2001) Calcium and bone metabolism in pregnancy and lactation. J Clin Endocrinol Metab 86, 2344-2348.

45. Islam MZ, Lamberg-Allardt C, Kärkkäinen M, et al. (2003) Dietary calcium intake in premenopausal Bangladeshi women: do socio-economic or physiological factors play a role? Eur J Clin Nutr 57, 674-680.

46. Kabir ML, Rahman M, Talukder K, et al. (2004) Rickets among children of a coastal area of Bangladesh. Mymensingh Med J 13, 53-58.

47. Greer FR (2008) 25-Hydroxyvitamin D: functional outcomes in infants and young children. Am J Clin Nutr 88, 529S-533S.

48. Kuroda E, Okano T, Mizuno N, et al. (1981) Plasma levels of 25-hydroxyvitamin $\mathrm{D}_{2}$ and 25-hydroxyvitamin $\mathrm{D}_{3}$ in 
maternal, cord and neonatal blood. J Nutr Sci Vitaminol (Tokyo) 27, 55-65.

49. Kratz A \& Lewandrowski KB (1998) Case records of the Massachusetts general hospital. Weekly clinicopathological exercises. Normal reference laboratory values. $N$ Engl J Med 339, 1063-1072.

50. Namgung R, Tsang RC, Specker BL, et al. (1994) Low bone mineral content and high serum osteocalcin and 1,25-dihydroxyvitamin D in summer- versus winter-born newborn infants: an early fetal effect? J Pediatr Gastroenterol Nutr 19, 220-227.

51. Dibba B, Prentice A, Laskey MA, et al. (1999) An investigation of ethnic differences in bone mineral, hip axis length, calcium metabolism and bone turnover between west African and Caucasian adults living in the United Kingdom. Ann Hum Biol 26, 229-242.

52. Silbergeld EK (1991) Lead in bone: implications for toxicology during pregnancy and lactation. Environ Health Perspect 91, 63-70.
53. Fischer PR, Rahman A, Cimma JP, et al. (1999) Nutritional rickets without vitamin D deficiency in Bangladesh. $J$ Trop Pediatr 45, 291-293.

54. Leffelaar ER, Vrijkotte TG \& van Eijsden M (2010) Maternal early pregnancy vitamin D status in relation to fetal and neonatal growth: results of the multi-ethnic Amsterdam born children and their development cohort. Br J Nutr 104, $108-117$.

55. Mahomed K \& Gulmezoglu AM (2000) Vitamin D supplementation in pregnancy. The Cochrane Database of Systematic Reviews, issue 2, CD000228.

56. Morley R, Carlin JB, Pasco JA, et al. (2006) Maternal 25hydroxyvitamin D and parathyroid hormone concentrations and offspring birth size. J Clin Endocrinol Metab 91, 906-912.

57. Viljakainen HT, Saarnio E, Hytinantti T, et al. (2010) Maternal vitamin $\mathrm{D}$ status determines bone variables in the newborn. J Clin Endocrinol Metab 95, 1749-1175. 